ISABELA MORBACH MACHADO E SILVA

\title{
DIREITO FINANCEIRO E OS FUNDOS DE EXPLORAÇÃO DE RECURSOS NATURAIS NÃO RENOVÁVEIS
}

\author{
Dissertação de Mestrado \\ Orientador: Prof. Associado Fernando Facury Scaff
}

FACULDADE DE DIREITO DA UNIVERSIDADE DE SÃO PAULO

SÃO PAULO - SP 


\section{DIREITO FINANCEIRO E OS FUNDOS DE EXPLORAÇÃO DE RECURSOS NATURAIS NÃO RENOVÁVEIS}

Dissertação apresentada à Banca Examinadora do Programa de Pós-Graduação em Direito, da Faculdade de Direito da Universidade de São Paulo, como exigência parcial para obtenção do título de Mestre em Direito, na área de concentração Direito Econômico e Financeiros, sob a orientação do Prof. Associado Dr. Fernando Facury Scaff.

FACULDADE DE DIREITO DA UNIVERSIDADE DE SÃO PAULO

SÃO PAULO 
Machado e Silva, Isabela Morbach

Direito Financeiro e os fundos de exploração de recursos naturais não renováveis / Isabela Morbach Machado e Silva ; orientador Fernando Facury Scaff -- São Paulo, 2017. $190 \mathrm{fl}$.

Dissertação (Mestrado - Programa de Pós-Graduação em Direito Direito Econômico, Financeiro e Tributário) - Faculdade de Direito, Universidade de São Paulo, 2017.

1. Direito Financeiro. 2. Fundos. 3. Fundos Soberanos de Riqueza. 4. Recursos naturais não renováveis. I. Scaff, Fernando Facury, orient. II. Título. 


\title{
DIREITO FINANCEIRO E OS FUNDOS DE EXPLORAÇÃO DE RECURSOS NATURAIS NÃO RENOVÁVEIS
}

\begin{abstract}
Dissertação apresentada à Banca Examinadora do Programa de Pós-Graduação em Direito, da Faculdade de Direito da Universidade de São Paulo, como exigência parcial para obtenção do título de Mestre em Direito, na área de concentração Direito Econômico e Financeiro, sob a orientação do Prof. Associado Dr. Fernando Facury Scaff.
\end{abstract}

Aprovado em:

BANCA EXAMINADORA:

Professor Associado Fernando Facury Scaff (Orientador)

Instituição: Faculdade de Direito da Universidade de São Paulo

Assinatura:

Professor(a):

Instituição: Faculdade de Direito da Universidade de São Paulo

Assinatura:

Professor(a):

Instituição:

Assinatura: 


\section{AGRADECIMENTOS}

Enquanto escrevia este trabalho revisitei cada uma das disciplinas que tive a oportunidade de cursas no período em que me dediquei ao mestrado. Me aventurei naquelas mais próximas à minha área de estudo, o Direito, e também em outras áreas do conhecimento. Concluída esta dissertação, posso afirmar, sem dúvidas, que há aqui fragmentos das lições aprendidas em todas as salas de aula frequentadas nos últimos três anos. Nunca conseguirei retribuir diretamente a paciência e os ensinamentos dispensados, por isso, o mínimo que me cabe é deixar registrado nominalmente cada professor com quem tive a oportunidade de conviver em sala de aula.

Agradeço especialmente ao meu orientador, professor Fernando Facury Scaff pela orientação e dedicação, pelas ideias trocadas e os muitos cafés que fizeram parte desta jornada. Obrigada por ter acreditado e me escolhido como sua orientanda.

Aos professores desta faculdade, José Maurício Conti, Gilberto Bercovici, Luis Fernando Massonetto, Regis Fernandes de Oliveira, Ana Maria Nusdeo, Heleno Taveira Torres, Diogo Rosenthal Coutinho, Virgílio Afonso da Silva, Jean-Paul Rocha, Conrado Hübner Mendes e Floriano de Azevedo Marques Neto.

Muito obrigada também aos professores de outras áreas que abriram as portas de suas salas cientes de minhas limitações, especialmente aos professores Basilia Maria Baptista Aguirre e Alexandre Macchione Saes, da Faculdade de Economia (USP); à professora Marta Arretche, da Faculdade de Ciência Política (USP), à professora Hirdan Katarina de Medeiros Costa do Instituto de Energia e Meio Ambiente (USP).

Como não poderia deixar de ser, gostaria de agradecer também a todos aqueles que de forma direta ou indireta me ajudaram na concretização deste trabalho. Há sempre uma sensação que nos persegue, ao escrever agradecimentos, deixar de mencionar alguém a quem devemos menção. Se o fiz, me desculpo desde já.

Aos que nunca hesitaram em apoiar minha decisão de vir para São Paulo estudar, me encorajaram - quase diariamente -, a seguir minhas convicções e a acreditar que seria capaz de concluir esta etapa. Tenho uma sorte tremenda de ter as melhores pessoas ao meu lado.

À minha mãe, Mirtes, eu não seria capaz de desejar mais amor, dedicação e apoio do que recebo. Não conheço ninguém mais digna da minha admiração. 
Ao meu pai, Claudio, a incondicional confiança que sempre depositou em mim, com o amor, a leveza, dedicação e generosidade tão características do papai, tornou essa trajetória sem dúvida mais leve e até possível.

Ao meu paidrasto, Flávio, por ter insistido dez anos atrás para que eu não seguisse o caminho mais fácil. Parte dos meus méritos são seus.

Agradeço também a minha gigante família "Machado e Silva-Morbach-Nassar", aqui representada por Juliana, Lucas e Ana Carolina.

Por tornar São Paulo um lugar mais acolhedor, Lucas, Malu, Raphael, Ana Carolina, Marina, Daniela, Laís, João, Alexandre, Bruna, Ana Clara, Fernanda, Carolina, Bárbara e Maria Virginia. Essa cidade faz muito mais sentido com vocês por perto.

Por manter Belém do Pará minha referência de porto seguro, de minha casa, lugar aonde estão meus mais preciosos amigos, me dando a tranquilidade necessária para desbravar o mundo com a certeza de poder retornar, Izabela Sauma e Lia Freire, vocês representam essa segurança e todos os amigos que não posso citar.

Esse trabalho não teria sido possível sem o auxílio e a disposição de Alexandre Silveira, Luísa Weichert, Daniel Athias, Alexandre Ramôa e Lucas Morbach., cuja calma e boa vontade sempre serão lembrados. 
"A estrada da vida é uma reta marcada de encruzilhadas. Caminhos certos e errados, encontros e desencontros do começo ao fim. Feliz aquele que transfere o que sabe e aprende o que ensina" (Cora Coralina, Exaltação de Aninha 'O professor')

\section{E então...}

"O tempo está maduro, é já o fim do futuro" (Flávio Nassar, O armagedon na cidade do Pará)

E assim nos foi dado viver, Nesse eterno recomeçar. 


\section{Resumo}

Junto à expectativa de aumento de receitas decorrentes da exploração de petróleo e gás após o descobrimento da camada pré-sal brasileira, surgiram importantes questionamentos sobre a melhor forma de administrar as receitas pública em prol do interesse público presente $\mathrm{e}$ também o das futuras gerações. Nesse contexto, o Poder Executivo encaminhou ao Congresso Nacional uma proposta de marco regulatório para a formulação e implementação das políticas públicas no setor energético. Como parte dessa nova política, foi criado o Fundo Social do petróleo, um Fundo Soberano de Riqueza com a finalidade de constituir fonte de recursos para o desenvolvimento social e regional, na forma de programas e projetos nas áreas de combate à pobreza e de estímulo ao desenvolvimento. Nesse contexto, o presente trabalho se propõe a analisar Fundo Social brasileiro, a partir de algumas experiências de outros países proprietários de Fundos Soberanos de Riqueza: Noruega, Chile, Botswana, Alberta (Canadá) e Alaska (Estados Unidos da América). Observar-se-á nas experiências selecionadas os possíveis erros e acertos que possam contribuir como lição para melhor regulamentação do fundo brasileiro.

Palavras-chave: Direito Financeiro, recursos naturais não renováveis, Fundo Social e Fundo Soberano de Riquezas 


\begin{abstract}
Alongside expectations of an increase in revenue from the exploration of oil and gas after the discovery of Brazil's pre-salt layer, important issues emerged on the best methods of administering public revenue in the benefit of current public interest and futures generations. In this context, the Executive Branch sent to the National Congress a proposal for a new regulatory framework for the formulation and implementation of public policies in the energy sector. As part of this policy a Social Fund of petroleum and a Sovereign Wealth Fund were created in order to create a source of resources for social and regional development, though programs and projects aimed at combating poverty and stimulating development. In light of this, this dissertation aims to analyze Brazil's Social Fund from experiences in other countries who also have Sovereign Wealth Funds: Norway, Chile, Botswana, Alberta (Canada) and Alaska (United States of America). It is possible to observe, through these selected experiences, possible hits and misses that may contribute as lessons to improve Brazil's fund regulation.
\end{abstract}

Key words: Public Finance Law, non-renewable natural resources, Social Fund and Sovereign Wealth Fund. 


\section{LISTA DE ABREVIATURAS}

AHSTF Alberta Heritage Savings Trust Fund

AIMCo Alberta Investment Management Corporation

APF Alaska Permanent Fund

APFC Alaska Permanent Fund Corporation

BoB Bank of Botswana

CDFS Conselho Deliberativo do Fundo Social

CGFFS Comitê de Gestão Financeira do Fundo Social

CID Canada Investment Division

CODELCO Corporación Nacional de Cobre del Chile

CONCLA Comissão Nacional de Classificação

CNP Conselho Nacional de Petróleo

CPD Capital Projects Divison

EID Energy Investment Division

FEES Fondo de Estabilización Económica y Social

FMI Fundo Monetário Internacional

FPM Fundo de Participação dos Municípios

FRP Fondo de Reserva de Pensiones

FS Fundo Social

FSR Fundos Soberanos de Riqueza

GPFG Government Pension Fund Global

GRF General Revenue Fund

GTREL Grupo Técnico de Padronização de Relatórios

IDH Índice de Desenvolvimento Humano

IFSWF International Forum of Sovereign Wealth Funds 
IWG International Working Group of Sovereign Wealth Funds

LDO Lei de Diretrizes Orçamentárias

LOA Lei Orçamentária Anual

LRF Lei de Responsabilidade Fiscal

NBIM Norges Investiment Bank Management

OECD Organisation for Economic Co-operation and Development

ONU Organização das Nações Unidas

OPEP Organização dos Países Exportadores de Petróleo

PIB Produto Interno Bruto

PF Pula Fund

PPA Plano Plurianual

PPSA Empresa Brasileira de Administração de Petróleo e Gás Natural S.A. - PréSal Petróleo S.A.

RNNR Recursos Naturais Não Renováveis

STN Secretaria do Tesouro Nacional 


\section{LISTA DE FIGURAS}

Figura 1 Número de Fundos Soberanos de Riqueza ............................................. 41

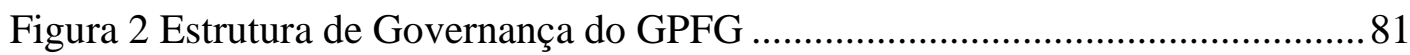

Figura 3 Tipos de Investimento - Fundos da Noruega (março de 2015) ..................82

Figura 4 AHF Valor de Mercado ................................................................. 110

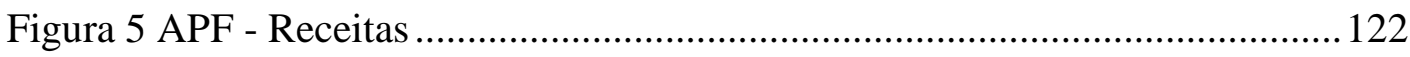

\section{LISTA DE GRÁFICOS}

Gráfico 1 Reserva de Óleo Cru - Noruega ......................................................... 71

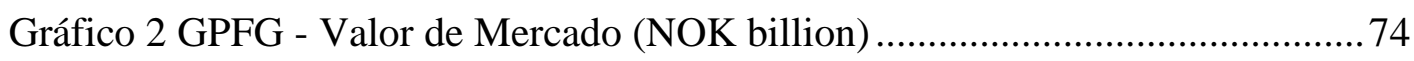

Gráfico 3 APF - Uso do retorno do Fundo......................................................... 124

Gráfico 4 APF - Alocação de Recursos (2009).................................................. 125

\section{LISTA DE TABELAS}

Tabela 1 BRASIL - Reserva Comprovada de Petróleo......................................... 145

Tabela 2 Receitas transferidas ao Fundo Social................................................... 147

Tabela 3 Percentual de repartição de royalties................................................. 150

Tabela 4 FSBR - Transferência de Receitas ........................................................... 153 


\section{SUMÁRIO}

INTRODUÇÃO. 16

1. O SURGIMENTO DE FUNDOS SOBERANOS E A EXPLORAÇÃO DOS RECURSOS NATURAIS NÃO RENOVÁVEIS ......................................................20

1.1. Os recursos naturais não renováveis e seus efeitos na economia ...........20

1.1.1. Recursos naturais: definição e importância. ........................................... 20

1.1.2. Recursos naturais não renováveis .......................................................2 22

1.1.3. O paradoxo da abundância dos recursos naturais .................................. 25

1.1.4. O paradoxo dos recursos naturais é uma maldição? ............................... 35

1.2. Fundos Soberanos como mecanismo de proteção à Maldição dos

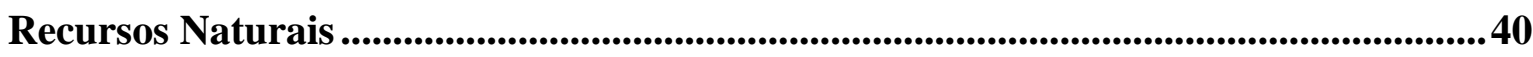

1.2.1. Origem e definição....................................................................... 40

1.2.2. O que são e qual a sua finalidade........................................................ 48

1.2.3. Enquadramento e base jurídico-legal.................................................52

1.2.4. Princípios de Santiago e as preocupações globais .................................54

2. FUNDOS SOBERANOS - EXPERIÊNCIAS INTERNACIONAIS.........64

2.1. Critérios de seleção ......................................................................64

2.2. Government Pension Fund - Global (GPFG) da Noruega ....................68

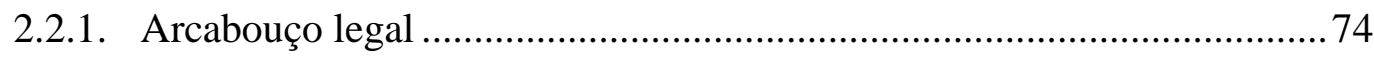

2.2.2. Fonte de financiamento e composição de receitas .................................. 76

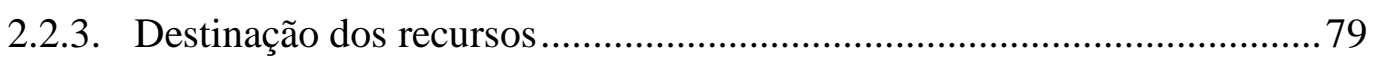

2.2.4. Governança, transparência e controle …................................................. 80

2.2.5. Política de Investimento dos ativos .................................................... 82

2.3. Chile: Fondo de Estabilización Económica y Social (FEES) ..................83

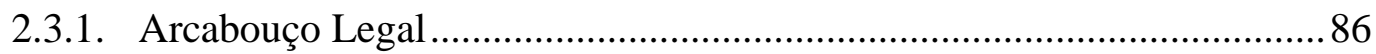

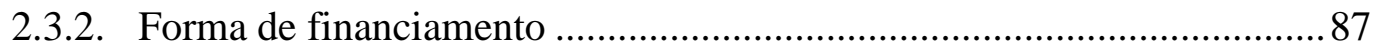




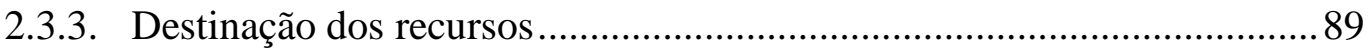

2.3.4. Governança, transparência e controle .................................................... 90

2.3.5. Política de Investimento...................................................................... 92

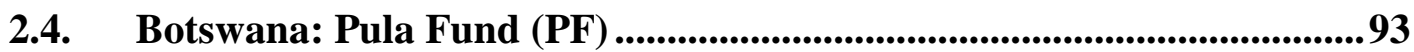

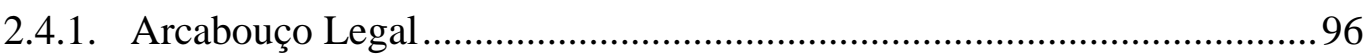

2.4.2. Forma de Financiamento e regras de retiras de recursos ........................97

2.4.3. Governança, transparência e controle ................................................. 100

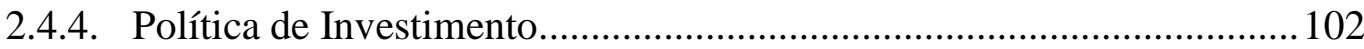

\subsection{Alberta Heritage Savings Trust Fund (AHSTF) - Alberta, Canadá .. 103}

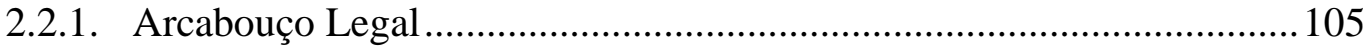

2.2.2. Fonte de financiamento e composição de receitas ................................. 106

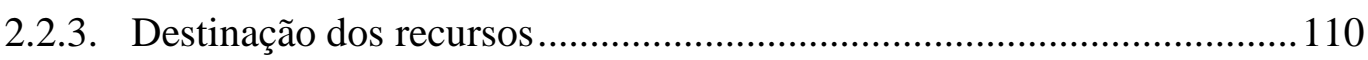

2.5.1. Governança, transparência e controle ................................................ 114

2.2.4. Política de Investimento dos ativos .....................................................116

2.6. Alaska: Alaska Permanent Fund (APF) ..............................................117

2.6.1. Arcabouço Legal ................................................................................ 119

2.6.2. Fonte de financiamento e composição de receitas ................................. 120

2.6.3. Destinação dos recursos ..................................................................... 123

2.6.4. Governança, transparência e controle ................................................ 124

2.6.5. Política de Investimento dos ativos .................................................... 125

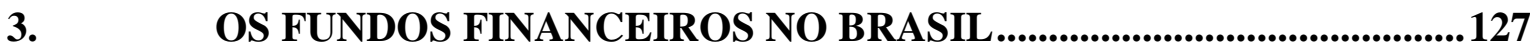

3.1.1. A trajetória jurídica relativa aos Fundos Públicos brasileiros ............... 127

3.1.2. Origem e Definição dos Fundos no ordenamento jurídico brasileiro .... 129

3.1.3. Natureza e personalidade jurídica....................................................... 134

3.1.4. Fundos em Espécie ........................................................................... 136

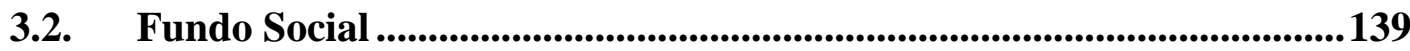

3.2.1. Estado e petróleo no Brasil ..................................................................... 139

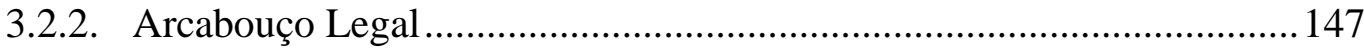

3.2.3. Fonte de Financiamento e composição de receita ................................ 149

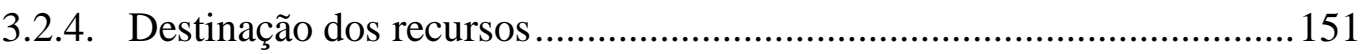


3.2.5. Governança, transparência e controle .................................................. 154

3.2.6. Fundo Social em perspectiva comparativa ......................................... 156

CONCLUSÕES......................................................................................................161

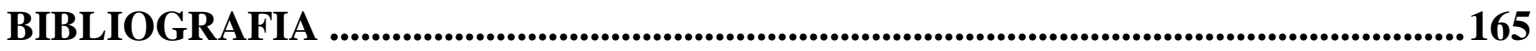




\section{INTRODUÇÃO}

Com a descoberta de grandes reservas de petróleo e gás natural na camada pré-sal em 2007, ${ }^{1}$ acentuou-se a expectativa por uma tendência de aumento de produção e exportação dessas commodities no Brasil. Consequentemente, importantes questionamentos ressurgiram acerca dos impactos econômicos que a produção causaria (e ainda causará); bem como acerca dos rumos das políticas econômicas, financeiras e sociais a serem adotadas.

Entre as questões importantes a serem respondidas, Giovani Loss aponta algumas: (1) serão privilegiados problemas sociais e econômicos de curto prazo ou de longo prazo?; (2) quais serão os melhores instrumentos para proteger o país contra flutuações de preços e da futura escassez desses recursos?; (3) como garantir que as rendas governamentais decorrentes da exploração dessas reservas serão utilizadas em prol do interesse público e não de interesses privados ${ }^{2}$. Ademais, Sérgio Gobetti ${ }^{3}$ questiona (4) o que faremos com os recursos?; (5) quando e como vamos gastar?; (6) como evitaremos os percalços sofridos por outros países?; e como garantir que os recursos decorrentes da exploração no presente alcancem as gerações futuras?

Diante da possibilidade de mudança de status do Brasil para figurar como uma das maiores reservas de petróleo e gás do mundo, o Poder Executivo viu-se obrigado a levar ao Poder Legislativo a discussão dos rumos do petróleo e tentar definir uma nova forma de alocar os recursos dele decorrentes. Era necessário discutir quais objetivos e metas deveriam ser alcançadas, traçando políticas públicas e planejamentos econômicos, que levem em consideração os benefícios e malefícios da atividade extrativa, observando outros países produtores e casos de ocorrência do fenômeno da Maldição dos Recursos.

1 LUNA, Denise e KHALIP, Andrei. Petrobras descobre reserva gigante de petróleo; ações disparam. REUTERS. 08 nov. $2007 . \quad$ Disponível http://noticias.uol.com.br/ultnot/reuters/2007/11/08/ult27u63482.jhtm

2 LOSS, Giovani R. Dutch Disease e os Fundos Soberanos de Petróleo e Gás. In: ARAGÃO, Alexandre Santos de. Direito do Petróleo e de outras fontes de Energia. Rio de Janeiro: Lumen Juris, 2011. p. 117

3 GOBETTI, Sérgio Wulff. Política e Pré-Sal: como gerir as rendas do petróleo e sustentar o equilíbrio macrofiscal no Brasil. XIV Prêmio Tesouro Nacional 2009, outubro/2009. p. 5. 
Nesse contexto, o Poder Executivo encaminhou ao Congresso Nacional uma proposta de marco regulatório, formado por quatro projetos de lei, os quais deram origem às Leis $n^{\circ}$ s $12.276 / 2010^{4}, 12.304 / 2010^{5}$ e $12.351 / 2010^{6}$. A proposta trouxe três principais inovações para a formulação e implementação das políticas públicas no setor energético: (i) Estabelecia novo regramento para a exploração do petróleo na área do pré-sal - o regime de partilha de produção; (ii) foi criada nova empresa pública chamada Empresa Brasileira de Administração de Petróleo e Gás Natural S.A. - Pré-Sal Petróleo S.A. (PPSA), responsável pela gestão dos contratos de partilha de produção e comercialização de petróleo e gás na área do pré-sal, e, ainda, (iii) foi instituído o Fundo Social, de natureza contábil e financeira, diretamente vinculado à Presidência da República.

No que tange ao item (iii), objeto do presente trabalho, destaca-se que o Fundo Social, em linhas gerais, foi criado com a finalidade de constituir fonte de recursos para o desenvolvimento social e regional, na forma de programas e projetos nas áreas de combate à pobreza e de estímulo ao desenvolvimento ${ }^{7}$, além de constituir poupança pública de longo prazo com base nas receitas auferidas pela União e, por fim, visando também mitigar as flutuações de renda e de preços na economia nacional, decorrentes das variações na renda geradas pelas atividades de produção e exploração de petróleo e de outros recursos não renováveis.

Os Fundos Soberanos de Riqueza $(\mathrm{FSR})^{8}$, dentre os quais se insere o Fundo Social do petróleo e gás, são instrumentos financeiros comumente utilizados na implementação de

4 Autoriza a União a ceder onerosamente à Petróleo Brasileiro S.A. - PETROBRAS o exercício das atividades de pesquisa e lavra de petróleo, de gás natural e de outros hidrocarbonetos fluidos de que trata o inciso I do art. 177 da Constituição Federal, e dá outras providências.

5 Autoriza o Poder Executivo a criar a empresa pública denominada Empresa Brasileira de Administração de Petróleo e Gás Natural S.A. - Pré-Sal Petróleo S.A. (PPSA) e dá outras providências.

6 Dispõe sobre a exploração e a produção de petróleo, de gás natural e de outros hidrocarbonetos fluidos, sob o regime de partilha de produção, em áreas do pré-sal e em áreas estratégicas; cria o Fundo Social - FS e dispõe sobre sua estrutura e fontes de recursos; altera dispositivos da Lei nº 9.478, de 6 de agosto de 1997; e dá outras providências.

7 Caput do Art. 47 da Lei $n^{\circ} 12.351 / 2010$.

8 Fundos Soberanos também podem ser referidos na literatura como Fundos Soberanos de Riqueza e Fundos Soberanos de Investimentos. 
política de gestão de recursos oriundos da exploração de recursos naturais. Esses instrumentos têm a finalidade de sanar os problemas criados pelo grande volume de recursos injetados na economia, evitar os efeitos nefastos da Maldição dos Recursos, custear o desenvolvimento socioeconômico da geração presente e reservar recursos para as gerações futuras. Naturalmente, a implementação desses instrumentos no Brasil deve considerar as complexidades socioeconômicas existentes, tendo em vista que a maioria dos países proprietários de fundos soberanos possuem realidades muito distintas.

Um dos desafios centrais do legislador ao criar o Fundo Social deve ser projetar um arcabouço jurídico capaz de resguardar os objetivos do fundo contra mudanças discricionárias promovidas pelo Poder Legislativo - vez que valores não devem ser utilizados para socorrer eventuais problemas financeiros, como cobrir déficits orçamentários - e, ao mesmo tempo, garantir margem de manobra na tomada de decisões que necessitem de respostas rápidas.

É a análise do Fundo Social brasileiro o objetivo central desta dissertação, a partir da experiência de outros países proprietários de Fundos de recursos naturais não renováveis. Observar-se-á em algumas experiências selecionadas os possíveis erros e acertos que possam contribuir como lição para melhor regulamentação do fundo brasileiro.

A estrutura que se propõe para o desenvolvimento do trabalho é composta por esta introdução, seguida de três capítulos e conclusão.

O primeiro capítulo pode ser dividido em duas partes. A primeira tem como objetivo descrever os aspectos econômicos relacionados à riqueza de recursos naturais não renováveis, com foco direcionado à exploração de petróleo, gás e minérios, seus efeitos econômicos positivos e negativos. Considerando que é comumente aceito que as potências ricas em recursos naturais tendem a falhar no quesito crescimento e desenvolvimento de suas economias - principalmente as menos desenvolvidas - , em razão dos vários efeitos adversos da abundância de recursos - tais como a Maldição dos Recursos, a Doença Holandesa e o efeito carona -, o seu arcabouço teórico será referenciado, para situar a criação dos Fundos Soberanos de Riqueza em um contexto mais amplo e crítico.

A segunda parte do primeiro capítulo tem como ponto central os Fundos Soberanos, desde sua origem, passando pela busca de uma definição, os arcabouços jurídicos mais 
comuns, a classificação internacional proposta pelo Fundo Monetário Internacional (FMI), até as preocupações por trás da expansão desses instrumentos ao redor do mundo.

No capítulo dois são apresentados cinco fundos decorrentes da exploração de recursos naturais não renováveis escolhidos, contemplando os seguintes aspectos: origem e contexto de sua criação, objetivo, arcabouço legal, suas fontes de financiamento (receitas que compõem o fundo), a destinação dos recursos, governança e transparência, e política de investimento. A análise não se pretende meramente descritiva. Demonstrar-se-á sua pertinência em relação à conjuntura política e econômica em que se situa, apontando suas peculiaridades e razões de ser. O objetivo do capítulo é apresentar da forma clara os aspectos comuns e em que ponto divergem as experiências de utilização dos fundos em suas diversas finalidades.

No último capítulo é apresentada a estrutura jurídica brasileira relativa aos fundos financeiros, seus diferentes arranjos institucionais, e de que forma o Fundo Social se insere no ordenamento jurídico financeiro do país. Na sequência, será apresentada a atual estrutura de funcionamento, vinculando-a com as informações apresentadas nos capítulos anteriores, de maneira a identificar os seus aspectos positivos e negativos, para propor contribuições à ainda recente experiência nacional.

Os principais debates conduzidos durante os demais capítulos serão analisados, ressaltando as razões que neles predominaram. E então será apresentada a análise crítica do Fundo Social brasileiro em face dos demais fundos analisados, sugerindo os aspectos a serem observados, aperfeiçoados ou corrigidos, sobre a estrutura jurídica e institucional em razão da experiência internacional.

Por fim, são apresentadas as conclusões. 


\section{CONCLUSÕES}

Os Fundos Soberanos de Riqueza (FRS) podem assumir papéis ou finalidades diversas. Entre suas aplicações, os FRS podem ser instrumentos fundamentais ao correto manejo da poupança das nações, que se veem expostas à volatilidade da economia internacional. Ou, ainda, como contrapontos aos efeitos colaterais do Paradoxo da Abundância ou da Maldição dos Recursos.

Vistos de outro ângulo, os fundos podem ainda representar a busca pela promoção da equidade intergeracional, preocupação que deveria nortear a exploração dos recursos naturais não renováveis.

Para alcançar tais finalidades, os FSRs geralmente procuram, ao lado de outros instrumentos macro e microeconômicos, (i) estabilização de curto prazo das receitas apuradas com a comercialização desses recursos; (ii) formação de poupança de longo prazo para futuras gerações, sem descuidar-se da (iii) promoção hodierna do desenvolvimento econômico e social, questões que impõem desafios consideráveis à administração de tais instrumentos públicos.

Deste modo, no decorrer das análises aqui empreendidas, sobre cada fundo eleito e analisado, algumas dessas características receberam destaques. Entre as mais importantes considerou-se o grau de representatividade das receitas decorrentes da exploração dos recursos naturais não renováveis em relação às receitas totais do ente, ou seja: país ou estado; e ainda a disciplina com que o sistema jurídico-político trata o desenvolvimento, o fortalecimento e a manutenção de tais fundos.

Em países em que a relação entre a receita de exploração dos recursos naturais renováveis e a receita total não chega a ser expressiva, a relevância da constituição e manutenção de um Fundo Soberano de Riqueza é menor. Por outro lado, a ausência de dependência em âmbito nacional não significará que entes subnacionais não possam ser, apenas eles, deveras dependentes. São exemplos de dependência subnacional os casos do Alasca (EUA), Alberta (Canadá) e Rio de Janeiro (Brasil). Assim, tem mais apelo, ou mais sentido, a constituição de um Fundo vinculado ao ente subnacional, sempre que a dependência estiver concentrada naquele ente. Caso contrário, espera-se que pelo menos 
alguma parte das políticas do fundo nacional do país esteja direcionada àquela localidade ou região.

Neste item, voltamos os olhos à estrutura brasileira. Como observado no Capítulo 3, o Fundo Social brasileiro é de fato um Fundo Soberano de Riqueza, suas finalidades são mistas, combinando funções de fundo de estabilização e de fundo de poupança intergeracional. É de propriedade exclusiva da União, na condição de pessoa jurídica de direito público interno, os estados e municípios não participam de seu domínio ou gestão.

Essa circunstância, ou seja, a titularidade, parece caminhar na direção contrária aos exemplos analisados no Capítulo 2. É que a União, ente central, não deve ser considerada altamente dependente em termos de receitas de petróleo e minerais. Por outro lado, há estados e municípios brasileiros dependentes (i.e., Campos dos Goytacazes/RJ ou o próprio Estado do Rio de Janeiro) que não criaram seus próprios fundos nos períodos econômicos promissores, e suas finanças encontram-se em situação preocupante, para dizer o mínimo ${ }^{287}$.

O exemplo canadense é bastante instrutivo da importância e dos efeitos que o objetivo do fundo enseja. O Alberta Heritage Savings Trust Fund contribuiu para formar um grande estoque de bens públicos e para sustentar uma oferta de serviços públicos de excelente qualidade, fruto das décadas em que o fundo tinha como objetivo o desenvolvimento social e econômico de seus beneficiários. No entanto, o valor de mercado do fundo atualmente é relativamente pequeno, e a descoberta do petróleo no xisto americano ameaça gravemente o bem-estar social erigido sobre as areias betuminosas da província canadense.

Também é indispensável observar a disciplina fiscal. Mais do que a existência de uma legislação que regulamente minuciosamente os diversos aspectos relacionados aos fundos, o enforcement dessas regras é mais relevante que sua existência formal. É necessário que esteja bem estabelecido quais são as políticas de gestão e de investimento são preconizadas por seus responsáveis. Mais importante do que o sistema informar quem são

${ }^{287}$ Cristina Boeckel, Daniel Silveira, Henrique Coelho, Káthia Mello e Lívia Torres. Governo do RJ decreta estado de calamidade pública devido à crise. .Portal G1 de notícias, 16 jun. 2016. Disponível em: http://g1.globo.com/rio-de-janeiro/noticia/2016/06/governo-do-rj-decreta-estado-de-calamidade-publicadevido-crise.html 
os responsáveis por tais políticas, quais as sanções em caso de descumprimento, é tornar seu cumprimento cogente. Cabe sempre perquirir quais práticas de facto podem ser eficientes, responsáveis e benéficas do que o quadro legislativo sugere.

O principal exemplo da relevância que tem a disciplina para o tema em análise é o Pula Fund, de Botswana. A legislação relativa ao fundo é parca, quase inexistente. No entanto, observado o desenvolvimento do fundo ao longo dos anos, bem como a seriedade no cumprimento das normas de gestão e de uso dos recursos, decorrentes da exploração mineral, verifica-se que estão presentes estabilidade, qualidade e credibilidade institucionais.

Exemplo semelhante vem do fundo da Noruega para o qual, apesar da existência de regras formais de governança, boa parte da disciplina fiscal, relacionada às receitas de petróleo, não se encontra rigorosamente definida na legislação. E, apesar disso, (ou, quiçá, por conta disso), observa-se na prática que, na trajetória do CPFG, muitas vezes utilizam-se receitas abaixo do limite permitido, indicando que há disciplina fiscal e política que não esvaziam os recursos do fundo, mantendo-se sua acumulação e consecução de objetivos, ainda que, para isso, negue-se demandas conjunturais e até oportunistas.

Esse é o desafio que também se apresenta ao Brasil. As normas do FSB estão, em grande parte, estabelecidas. Mas isso não significa que a disciplina fiscal se faça presente. Há em nosso país o estranho fenômeno das leis que "pegam", em contraposição às leis que “não pegam”. São "para inglês ver" ${ }^{288}$. Tal comportamento parece não ter contribuído, ao longo do último século, para o desenvolvimento social e econômico do país. Ao contrário, criou-se um ambiente institucional de conveniência em detrimento da segurança jurídica e institucional, onde tudo pode desde que se detenha o poder para decidir.

288 A expressão tem origem em 1830, época em que a Inglaterra impôs ao Brasil o esforço de acabar com o tráfico de escravos através da edição de leis que desestimulassem tal prática. O Brasil acatou formalmente a exigência, aprovou a lei cientes de que não seria cumprida, daí que surgiram leis existentes somente "em um papel”, ou seja " leis para inglês ver". GURGEL, Algemiro Eloy. Uma lei para inglês ver: a trajetória da lei de 7 de novembro de 1831. Artigo disponibilizado na seção "Memorial do poder judiciário" no site do Tribunal de Justiça do Rio Grande do Sul. Disponível em: http://www.tjrs.jus.br/export/poder_judiciario/historia/memorial_do_poder_judiciario/memorial_judiciari o_gaucho/revista justica e historia/issn_1677-065x/v6n12/Microsoft_Word _ARTIGO_UMA_LEI_PARA_INGLXS_VER...._Argemiro_gurgel.pdf 
Merece ênfase, portanto, que os aparentemente níveis baixos de disciplina fiscal no Brasil - como é indicativa a alteração das regras de destinação dos valores do Fundo antes mesmo de sua criação - podem conduzir ao fracasso dos objetivos do FSB. Esta é uma das relevantes lições que se podem extrair da experiência internacional.

Dessa forma, e em busca de transformar essa realidade, há inúmeros estudos a empreender e conduzir no Direito e nas demais Ciências Sociais para fortalecer a estrutura dos FSB - são exemplos desses esforços, o estudo das diferentes possibilidades de modelagem jurídica de instituições; a escolha dos instrumentos mais adequados (conforme os fins a serem perseguidos); as diferentes formas de indução ou recompensa para adoção de comportamentos; a escolha dos tipos de normas utilizadas em relação a seu conteúdo e extensão; e o enforcement das normas. São todos exemplos de variáveis a considerar no aperfeiçoamento das instituições, como elementos substanciais ao mecanismo de promoção do desenvolvimento social e econômico.

A análise dos fundos aqui realizada parece indicar que o componente político - a disposição dos agentes em agir de acordo com um fim estabelecido - é tão ou quase tão importante quanto a estrutura jurídico-institucional em que se amparam os fundos. Nesse sentido, a análise da conjuntura política brasileira impõe uma escolha difícil: um desenho institucional que garanta flexibilidade ao gestor do fundo otimiza sua atuação e a possibilidade desses gestores utilizarem dessa discricionariedade de maneira irresponsável. Nesse contexto, a baixa disciplina fiscal, e mesmo a disparidade dos projetos políticos em disputa no Brasil - torna difícil afirmar que este ou aquele partido vá seguir uma política de curto ou longo prazo -, potencializam o aspecto da vulnerabilidade do Fundo Social.

Entende-se que o direito positivo pode ser um meio para formulação de políticas sociais e econômicas, determinando o que devem ser, repartindo competências e equilibrando as relações de poder. Mas legislar não é suficiente. É necessária a adoção de mecanismos que permitam a manter-se das normas editadas. Para tanto são indispensáveis o provimento dos meios de fiscalização de seu cumprimento e a correspondente punição por descumprimentos. 


\section{BIBLIOGRAFIA}

ACEMOGLU, Daron e ROBINSON, James A. Why Nations Fail: the origins of power, prosperity, and poverty. New York: Crown Business, 2012.

; JOHNSON Simon; and ROBINSON, James A. The Colonial Origins of Comparative Development: An Empirical Investigation. American Economic Review, v. 91,2001 , p. 1369-1401

AL-HASSAN, Abdullah et al. Sovereign wealth funds: Aspects of governance structures and investment management. IMF Working Paper - Monetary and Capital Markets Department. 2013.

AGÊNCIA NACIONAL DE PETRÓLEO. Anuário Estatístico 2013. Em: http://www.anp.gov.br/?pg=66833 Último acesso em: 05 mai. 2014.

ALDCROFT, Derek Howard. The European Economy 1914-2000. $4^{\mathrm{a}}$ edição. London (UK): Routledge, 2001. p. 152-156.

ATKINSON, Giles; HAMILTON, Kirk. Savings, growth and the resource curse hypothesis. World Development, v. 31, n. 11, p. 1793-1807, 2003.

AUTY, Richard M. Natural resources, capital accumulation and the resource curse. Ecological economics, n. 61.4, 2007. pp. 627-634.

. Resource abundance and economic development. Oxford University Press, 2001. The political economy of resource-driven growth. European economic review 45.4, 2001. pp. 839-846.

Natural resource endowment, the state and development strategy. Journal of International Development, v. 9, n. 4, p. 651-663, 1997.

Sustaining development in mineral economies: the resource curse thesis. New York: Routledge, 1993.

AVENDAÑO, Rolando; SANTISO, Javier. Are sovereign wealth funds' investments politically biased? A comparison with mutual funds. United States: OECD, 2009. (Working Paper, n. 283). 
BACON, Robert e TORDO, Silvana. Experiences with Oil Funds: Institutional and Financial Aspects. Washington: Energy Sector Management Assistance Program (ESMAP). Report 321/06, jun. 2006.

BAHGAT, Gawdat. Sovereign Wealth Funds: Dangers and Opportunities. International Affairs. Royal Institute of International Affairs, 1944, v. 84, N. 6. Nov. 2008, pp. 1189-1204.

BERCOVICI, Gilberto. Direito Econômico do Petróleo e dos Recursos Minerais. São Paulo: Quarties Latin, 2011. Direito Econômico do Petróleo e dos Recursos Minerais. São Paulo: Quartier Latin, 2011.

BERNSTEIN, Shai; LERNER, Josh; SCHOAR, Antoinette. The investment strategies of sovereign wealth funds. The Journal of Economic Perspectives, v. 27, No. 2 Spring, 2013.

BIELSCHOWSKY, Ricardo. Pensamento econômico brasileiro: o ciclo ideológico do desenvolvimentismo. Rio de Janeiro: Contraponto, 2000.

O velho e o novo desenvolvimentismo. Publicado no dia 06 nov. 2012. Disponível em: http://novo.fpabramo.org.br/content/o-velho-e-o-novo-desenvolvimentismo Último acesso em: $18 \mathrm{dez} .2014$.

BLUNDELL-WIGNALL, Adrian; HU, Yu-Wei; YERMO, Juan. Sovereign wealth and pension fund issues. France: OECD, 2008. (Working Paper, n. 14).

BOLTON, Patrick; SAMAMA, Frederic w SLIGLITZ, Joseph E. Soverign Weath Funds and Long-Term Investing. New York: Columbia University Press, 2012.

BRESSER-PEREIRA, Luiz Carlos. Doença Holandesa e sua neutralização: uma abordagem ricardiana. Revista de Economia Política, 28, p. 47-71, 2007. O novo desenvolvimentismo e a ortodoxia convencional. In: São Paulo em perspectiva, v. 20, n. 3, p. 5-24. Julho/Setembro, 2006.

BRUNNSCHWEILER, Christa N. Cursing the blessings? Natural resource abundance, institutions, and economic growth. World Development, v. 36, 3, Elsevier, 2008. 
CARRIÓ, Genaro A. Notas sobre Derecho y Language. Buenos Aires: Abeledo-Perrot, 1973, p. 72.

CARVALHO, Getúlio. Petrobras: do monopólio aos contratos de risco. Rio de Janeiro: Forense-Universitária, 1977.

CASELLI, Francesco e MICHAELS, Guy. Do oil windfalls improve living standards? Evidence from Brazil. Cambridge: National Bureau of Economic Research, Dec. 2009.

CHANG, Ha-Joon. Institutions and Economic Development. Journal of Institutional Economics, v. 7, 2011, p. 473-498.

COHEN, B. J. Sovereign wealth funds and national security: the great tradeoff. International Affairs, v. 85, n. 4, p. 713-731, 2009.

COSTA, Hirdan Katarina de Medeiros. O Princípio da Justiça Intra e Intergeracional como elemento na destinação Das Rendas De Hidrocarbonetos: Temática Energética Critica na Análise Institucional Brasileira. Tese de doutorado. Instituto de Energia, Universidade de São Paulo. 2012.

COSTA, Leonardo da Silva Guimarães Martins da. Uma visão Sistêmica dos Fundos Federais. Texto para discussões $\mathrm{n}^{\circ}$ 007. Tesouro Nacional: 2012. Disponível em: <http://www3.tesouro.fazenda.gov.br/textos_discussao/downloads/td7.pdf $>$. Último acesso em: 10 mai. 2015.

CONTI, José Maurício. Federalismo Fiscal e Fundo de Participação. São Paulo: Juarez de Oliveira, 2001.

DAS, D. K. Sovereign-wealth funds: a new role for the emerging market economies in the world of global finance. International Journal of Development Issues, v. 7, n. 2, p. 8096, 2008. Disponível em:

ENRIQUEZ, Maria Amélia Rodrigues da Silva. Equidade intergeracional na partilha dos benefícios dos recursos minerais: a alternativa dos Fundos de Mineração. Revista Iberoamericana de Economia Ecológica v. 5: 61-73, 2006. Disponível em: http://www.redibec.org/IVO/rev5_05.pdf Último acesso em:

FERNANDES RÊGO, Andressa Guimarães Torquato. Uma análise acerca da classificação das receitas petrolíferas na lei orçamentária. In: CONTI, José Maurício 
e SCAFF, Fernando Facury (coord.). Orçamentos Públicos e Direito Financeiro. São Paulo: Revista dos Tribunais, 2011. p. 433-452.

GOBETTI, Sérgio Wulff. Federalismo Fiscal e Petróleo no Brasil e no mundo. Texto para discussão 1669. Rio de Janeiro: IPEA, out.2011. Disponível em: 03 mai. 2014.

GODARD, Olivier. A gestão integrada dos recursos naturais e do meio ambiente: conceitos, instituições e desafios de legitimação. Gestão de recursos naturais renováveis e desenvolvimento: novos desafios para a pesquisa ambiental. São Paulo: Cortez, v. 500, p. 201-265, 1997.

GOVERNMENT PENSION FUND ACT. October, 8th, 2012. Disponível em: https://www.regjeringen.no/contentassets/9d68c55c272c41e99f0bf45d24397d8c/gover nmentpensionfundact.pdf

GRIFFITH-JONES, Stephany; OCAMPO, José Antonio. Sovereign Wealth Funds: A Developing Country Perspective. Paper prepared for the workshop on Sovereign Wealth Funds organized by the Andean Development Corporation. Fevereiro, 2008. Disponível em: http://www.g24.org/sowf0308.pdf Acesso em

GTREL - Grupo Técnico de Padronização de Relatórios. Relatório do dia 29 de outubro de 2011. Disponível em http://www.tesouro.fazenda.gov.br/-/grupo-tecnico-depadronizacao-de-relatorios-gtrel

GYLFASON, Thorvaldur. Natural resources, education, and economic development. European Economic Review, v. 45, n. 4, p. 847-859, 2001.

GURGEL, Algemiro Eloy. Uma lei para inglês ver: a trajetória da lei de 7 de novembro de 1831. Disponibilizado no memorial do poder judiciário do Tribunal de Justiça do Rio Grande do Sul, através do link http://www.tjrs.jus.br/export/poder_judiciario/historia/memorial_do_poder_judiciario/ memorial_judiciario_gaucho/revista_justica_e_historia/issn_1677-

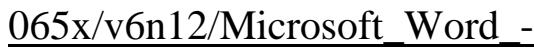
ARTIGO_UMA_LEI_PARA_INGLxS_VER.... Argemiro_gurgel.pdf 
HABER, Stephen H. MENALDO, Victor A. Do natural resources fuel authoritarianism? A reappraisal of the resource curse. American Political Science Review, v. 105, n. 1, 2010.

HOBSBAWN, Eric. A era dos extremos: o breve século XX. 1941-1991. São Paulo: Companhia das Letras, 1995.

HUMPHREYS, Macartan; SACHS, Jeffrey; STIGLITZ, Joseph (Orgs.). Escaping the Resource Curse. New York: Columbia University Press, 2007.

IIMI, Atsushi Escaping From The Resource Curse. IMF Staff Papers, n. 54, 2007, p. 663699.

IWG - International Working Group of Sovereign Wealth Funds. Sovereign Wealth Funds. Current institutional and operational practices. Disponível em: http://www.iwgswf.org/pubs/eng/swfsurvey.pdf Último acesso em: 01 abri 2016. . Sovereign Wealth Funds: Generally Accepted Principles and Practices “Santiago Principles”. October, 2008. Disponível em: http://www.iwgswf.org/pubs/eng/santiagoprinciples.pdf

INTERNATIONAL MONETARY FUND. A Multi-Donor Trust Fund for IMF. Capacity Building Technical Assistance in Managing Natural Resource Wealth Program Document. Revised. Nov. 2010. Disponível em: https://www.imf.org/external/np/otm/2010/110110.pdf Último acesso em: 05 jan. 2016.

Sovereign Wealth Funds - A work Agenda. 2008. Disponível em: http://www.imf.org/external/np/pp/eng/2008/022908.pdf Último acesso em:

JEN, Stephen. The definition of a sovereing wealth fund. Morgan Stanley Research Global, v. 26, oct. 2007.

KARL, Terry Lynn. The paradox of plenty: Oil booms and petro-states. University of California Press, 1997.

KERN, Steffen. Sovereign Wealth Funds - state investments on the rise. International Topics - Current Issues. Deutsche Bank Research, sep. 2007 
KIMMITT, Robert. M. Public footprints in private markets. Foreign Affairs, v. 87, n. 1, Jan/Fev 2008.

LARSEN, Erling Røed. Escaping the Resource Curse and the Dutch Disease? When and Why Norway Caught up withand Forged Ahead of Its Neighbors. American Journal of Economics and Sociology, v. 65, No. 3, Natural Resources, Taxation, and Regulation: Unusual Perspectives on a Classic Topic, jul. 2006. pp. 605-640.

LEDERMAN, Daniel; MALONEY, William F. (Ed.). Natural resources, neither curse nor destiny. World Bank Publications, 2006. 396p.

LEGISLATIVE COUNCIL SECRETARIAT. Fact Sheet. Government Pension Fund of Norway. FSC50/13-14. Disponível em: http://www.legco.gov.hk/researchpublications/english/1314fsc50-government-pension-fund-of-norway-20140902-e.pdf

LIE, Einar. Learning by failing. The origins of the Norwegian oil fund. Draft. University of Oslo, October-2013. Disponível em: http://eml.berkeley.edu// webfac/cromer/Lie.pdf LIMA, Paulo César Ribeiro. Pré-Sal, o novo marco legal e a capitalização da Petrobras. Rio de Janeiro: Synergia, 2011.

LOSS, Giovani R. Dutch Disease e os Fundos Soberanos de Petróleo e Gás. In: ARAGÃO, Alexandre Santos de. Direito do Petróleo e de outras fontes de Energia. Rio de Janeiro: Lumen Juris, 2011. P. 109-135.

MAGALHÃES, Andre Simas. Determinantes dos Fundos Soberanos de Investimentos e o Caso Brasileiro. Revista tempo do mundo-RTM, v. 3, n. 2, ago. 2011.

MAHDAVY, Hossein. The patterns and problems of economic development in rentier states: the case of Iran. Life, v. 1000, n. 1, 1970.

MAIPOSE, Gervaise S. Policy and institutional dynamics of sustained growth in Botswana. Commission on Growth and Development Working Paper, n. 35, 2008. Disponível em: http://www.unrisd.org/80256B3C005BCCF9/(httpAuxPages)/4365C57157F8EF16C1 257AEF00525641/\$file/Botswana\%20Maipose\%20web.pdf Último acesso em: 10 dez. 2016. 
MARINHO JÚNIOR, Ilmar Penna. Petróleo: política e poder. Rio de Janeiro: José Olímpio Editora, 1989.

MEHLUM, Halvor; MOENE, Karl; TORVIK, Ragnar. Institutions and the resource curse. The economic journal, v. 116, n. 508, p. 1-20, 2006.

NATAGA, Bruno Mitsuo. A limitação da discricionariedade em matéria orçamentária pelos princípios da legalidade, legitimidade e economicidade. In: CONTI, José Maurício e SCAFF, Fernando Facury (coord.). Orçamentos Públicos e Direito Financeiro. São Paulo: Revista dos Tribunais, 2011. p. 357-383.

NATURAL RESOURCE GOVERNANCE INSTITUTE. Norway Government Pension Fund Global. Disponível em: http://www.resourcegovernance.org/sites/default/files/NRF_Norway_July2013.pdf

NEUMAYER, Eric. Does the "Resource Curse"' hold for Growth in Genuine Income as Well? World Development, v. 32, No. 10, pp. 1627-1640, 2004.

NIELSEN, Lynge. Classifications of Countries based on their Level of Development: How it is Done and How it Could be Done. IMF Working Paper Strategy, Policy, and Review Department. 2011. Disponível em: https://pdfs.semanticscholar.org/bc7f/5b086b8db9adaa3f51645896fda5d1e46659.pdf Último acesso em: 01 dez. 2016.

OLIVEIRA, Regis Fernandes. Curso de Direito Financeiro, $3^{\mathrm{a}}$ ed. São Paulo: Revista dos Tribunais, 2010.

PAULANI, Leda. Acumulação sistêmica, poupança externa e rentismo: observações sobre o caso brasileiro. Estudos Avançados, 27 (77), 2013, pp. 237-261.

PEREIRA, Jesus Soares. Petróleo, energia elétrica e siderurgia: a luta pela emancipação. Rio de Janeiro: Paz e Terra, 1975.

PERMAN, Roger; MA, Yue; MCGILVRAY, James. Natural resource and environmental economics. New York (London): Longman, 1996.

PORTAL BRASIL. Linha do tempo: história do Petróleo. Disponível em: http://www.brasil.gov.br/linhadotempo/html/tema/lista_epocas?tema=Hist\%C3\%B3ria \%20do\%20Petr\%C3\%B3leo 
PREBISCH, Raúl. Crecimiento, desequilibrio y disparidades: interpretación del proceso de desarrollo económico. En: Estudio económico de América Latina, 1949-E/CN. 12/164/Rev. 1-1950-p. 3-89, 1950.

RAWLS, John. Uma teoria da Justiça. São Paulo: Martins Fontes, 1997.

REDIKER, Douglas; CREBO-REDIKER, Heidi. Foreign Investment and Sovereign Wealth Funds. New America Foundation - Global Strategic Finance Initiative. Working Paper, n. 1, sep. 2007. Disponível em: http://www.newamerica.net/files/GSFIWorkingPaper1.pdf. Último acesso em:

REIS, Cristina. Estrutura produtiva e instituições no desenvolvimento econômico a partir de Recursos Naturais: uma análise teórica e crítica. ANPEC-Associação Nacional dos Centros de Pós-graduação em Economia, 2014. Disponível em: https://www.anpec.org.br/encontro/2012/inscricao/files_I/i556cda81f3ee955d6769594f3830964cd.pdf Último acesso em: 20 out. 2016.

RIBEIRO, Elaine. Direito do Petróleo, Gás e Energia. Rio de Janeiro: Elsevier, 2010. RINALDI, Patrícia Nogueira. Estados e Fundos Soberanos de Riqueza: Instrumentos de retrocesso ou avanço da globalização econômica. Dissertação de Mestrado. Instituto de Filosofia e Ciências Humanas da Universidade Estadual de Campinas. 2010.

RODRIGUEZ, Jorge C. et al. Evolución, administración e impacto fiscal de los ingresos del cobre en Chile. Chile: Publicación de la Dirección de Presupuestos del Ministerio de Hacienda, jun. 2015.Disponível em: http://www.dipres.gob.cl/572/articles133158 doc_pdf.pdf . TOKMAN, Carla R.; VEGA, Alejandra C. Structural balance policy in Chile. OECD Journal on Budgeting, v. 7, n. 2, p. 59-92, 2007.

RODRIGUEZ, Francisco; SACHS, Jeffrey D. Why do resource-abundant economies grow more slowly? Journal of Economic Growth, v. 4, n. 3, p. 277-303, 1999.

ROZANOV, Andrew. From Reserves to Sovereign Wealth Management. Central Banking, Volume XV, Number 3, February 2005.

ROY, Sudip. The rulers of finance. London: Euromoney, dez. 2007. 
RUBINSTEIN, Flávio. Receitas públicas de recursos naturais no direito financeiro brasileiro. 2012. Tese de Doutorado. Faculdade de Direito da Universidade de São Paulo.

SACHS, Jeffrey D.; WARNER, Andrew M. The curse of natural resources. European Economic Review, v. 45, n. 4, p. 827-838, 2001. ; ___ Economic convergence and economic policies. National Bureau of Economic Research, n. w5039, 1995.

SANCHES, Osvaldo Maldonado. Fundos Federais: origens, evolução e situação atual na administração federal. Revista de Administração Pública. v. 36, n 4, p. 627-670. Rio de Janeiro, jul. 2002.

SCAFF, Fernando Facury. Royalties do Petróleo, Minério e Energia. Aspectos Constitucionais, Financeiros e Tributários. São Paulo: Revista dos Tribunais, 2014.

SCLIAR, Claudio. Geopolitica das minas do Brasil - a importância da mineração para a sociedade. Rio de Janeiro: Revan, 1996.

SERRA, Rodrigo Valente. Rendas petrolíferas no Brasil: critérios de distribuição distorcidos induzem ineficiência do gasto. In: MENDES, Marcos (org.). Gasto público eficiente - propostas para o desenvolvimento do Brasil. Rio de Janeiro: Top Books editora, 2006.

SIAS, Rodrigo. O fundo soberano brasileiro e suas implicações para a política econômica. Revista do BNDES, Rio de Janeiro, V. 15, N. 30, P. 93-127, Dez. 2008. P. 97

SINNOTT, Emily. John Nash y Augusto de la Torre: Recursos naturais na América Latina: indo além das altas e baixas. Rio de Janeiro: Elsevier, 2010

SILVA, Maria Amélia Rodrigues da. Economia dos recursos naturais. Economia do meio ambiente: teoria e prática. Rio de Janeiro: Elsevier, 2003.

SILVEIRA, Alexandre Coutinho. Governança pública de royalties: Federalismo fiscal e futuras gerações. Dissertação de Mestrado. Universidade de São Paulo, 2014.

SOUZA, Marcos Tadeu Napoleão de. O Fundo social e o PL $n^{o} 323$, de 2007. Consultoria Legislativa. Brasília, Câmara dos Deputados. Agosto/2013. 
SMITH, Stuart Landon and Constance. Government Revenue Stabilization Funds: Do They Make Us Better Off? Canadian Public Policy / Analyse de Politiques, v. 39, n. 01, mar. 2013, pp. 71-99.

SMITH, Roger S.. Income Growth, Government Spending, and Wasting Assets: Alberta's Oil and Gas. Canadian Public Policy / Analyse de Politiques, v. 18, No. 4. Dec., 1992, pp. 387-412.

TORRES, Heleno Taveira e RODRIGUES, Felipe da Cunha. Fundo Soberano do Brasil e Finanças Públicas Regime Jurídico dos fundos públicos especiais, experiência internacional e a Lei $n^{\circ}$ 11.887/2008. Belo Horizonte: Fórum, 2012.

TORRONTEGUY, Alessandra Folzke. A aplicação dos Royalties do Petróleo na efetividade dos direitos fundamentais. São Paulo: LTr, 2011.

TORVIK, Ragnar. The Political Economy of Reform in Resource Rich Countries. Norwegian University of Science and Technology. Drawn from the author's lecture at a high-level seminar on Natural resources, finance, and development: Confronting Old and New Challenges, organized by the Central Bank of Algeria and the IMF Institute in Algiers, on 4-5 November/2010. Disponível em: http://www.imf.org/external/np/seminars/eng/2010/afrfin/pdf/Torvik2.pdf Último acesso em:

TRUMAN, M. Edwin. Sovereign Wealth Funds: Threat or Salvation? Washington, DC: Peterson Institute for International Economics, sep. 2010.

WFG. SWFs generally accepted principles and practices. Santiago Principles, October, 2008. Disponível em: http://www.ifswf.org/santiago-principles Último acesso em: 05 jun. 2016.

VELHO, José Lopes. Petróleo: Dádiva e maldição. 150 anos de história. Lisboa: Bnomics, 2010. 419p.

VENTURI, Luis Antonio Bittar. Recurso Natural: A construção de um conceito. GEOUSP - Espaço e Tempo, São Paulo, n. 20, pp. 09 - 17, 2006.

VIEIRA, Fábio Alonso. Os fundos soberanos de riqueza como instrumento ao desenvolvimento integral. Belo Horizonte: Arraes, 2013. 
VIVACQUA, Attílio. A Nova Política do Subsolo e o Regime Legal das Minas. Rio de Janeiro: Editora Panamericana, 1942.

\section{Referências específicas sobre os fundos}

ABU DHABI INVESTMENT AUTHORITY WEBSITE. Disponível em: http://www.adia.ae/. Último acesso em:

ALASKA PERMANENT FUND CORPORATION WEBSITE. Disponível em: http://www.apfc.org/home/Content/home/index.cfm. Último acesso em:

ALBERTA INVESTMENT MANAGEMENT CORPORATION - AIMC. Annual Report. 2013. Disponível em: http://www.aimco.alberta.ca/AIMCo_AR_2014/AIMCo-2013Annual-Report.pdf Último acesso em: 10 mai. 2014.

ALASKA PERMANET FUND COMISSION. An Alaskan's Guide to the Permanent Fund. 12th Guide Edition, July/2009. Disponível em: http://www.apfc.org/home/Media/publications/2009AlaskansGuide.pdf Último acesso em:

ALASKA PERMANENT FUND CORPORATION. Annual Report, 2014. Em: http://www.apfc.org/_amiReportsArchive/APFC_2014.pdf Último acesso em: . Annual Report, 2013. Disponível em: http://www.apfc.org/_amiReportsArchive/FY2013AnnualReport.pdf Último acesso em: 30 abr. 2016.

. Annual Report, 2012. Disponível em:

http://www.apfc.org/_amiReportsArchive/FY2012AnnualReport.pdf Último acesso em:

Annual Report, 2011. Disponível em:

http://www.apfc.org/_amiReportsArchive/APFC\%20Annual\%20Report\%202011.pdf Último acesso em: 30 abr. 2016. 
Annual Report, 2010. Disponível em:

http://www.apfc.org/_amiReportsArchive/2010AnnualReport.pdf Último acesso em: 30 abr. 2016.

ALASKA PERMANENT FUND CORPORATION. Investment Policy. 10 dez. 2014.

Disponível em:

http://www.apfc.org/_amiReportsArchive/Investment\%20Policy\%20(12-10-

14\%20FINAL).pdf Último acesso em: 30 abr. 2016.:

ALASKA PERMANENT FUND CORPORATION. Board of Trustees Charters and Governance Policies. February/2014. Disponível em:

http://www.apfc.org/_amiReportsArchive/APFC\%20Governance\%20Manual\%20\%2027Feb2014.pdf Último acesso em:

ALBERTA, CANADÁ. Alberta Heritage Savings Trust Fund Act. Consolidado em 29 abril 2013. Disponível em: http://www.qp.alberta.ca/documents/Acts/A23.pdf Último acesso em:

ALBERTA, CANADÁ. Fiscal Management Act. Consolidado em 29 abril 2013.

Disponível em: http://www.qp.alberta.ca/documents/Acts/f14p5.pdf> Último acesso em:

ALBERTA ENERGY. Acquisitions of Petroleum and Natural Gas Rights in Alberta. Junho/2014. Disponível em:

http://www.energy.alberta.ca/LandAccess/pdfs/ERSfsEDUAtenure.pdf Último acesso em: 30 abr. 2016.

ALBERTA GOVERNMENT. Alberta Oil Sands Industry Quaterly Update. 2013.

Disponível em:

https://albertacanada.com/files/albertacanada/AOSID_Quarterly_Update_Summer201 3.pdf Último acesso em:

ALBERTA GOVERNMENT. Alberta's Oil Sands: The Facts. January, 2014. Disponível em: http://www.energy.alberta.ca/OilSands/pdfs/AlbertasOilSandsFactsJan14.pdf Último acesso em: 
ALBERTA HERITAGE SAVINGS TRUST FUND. Annual Report, 2012-2013.

Disponível em: http://www.finance.alberta.ca/business/ahstf/annualreports/2013/Heritage-Fund-2012-13-Annual-Report.pdf Último acesso em: $30 \mathrm{abr}$. 2016.

ALBERTA HERITAGE SAVINGS TRUST FUND. Annual Report, 2013-2014. Disponível em: http://www.finance.alberta.ca/business/ahstf/annualreports/2014/Heritage-Fund-2013-14-Annual-Report.pdf Último acesso em:

ALBERTA HERITAGE SAVINGS TRUST FUND. Business Plan, 2013-2016. Disponível em: http://www.finance.alberta.ca/publications/budget/budget2013/heritage-fund.pdf Último acesso em: 30 abr. 2016.

ALBERTA HERITAGE SAVINGS TRUST FUND. 3rd Quarter Report, 2014-2015. Disponível em: http://www.finance.alberta.ca/business/ahstf/quarterly-reports/20143rdq/Heritage-Fund-2014-15-3rd-Quarter-Report.pdf Último acesso em:

ALBERTA HERITAGE SAVINGS TRUST FUND. Heritage Fund Statement of Investment and Policy Goals. April, 2011. Disponível em: http://www.finance.alberta.ca/business/ahstf/heritage-fund-statement-investmentpolicy-and-goals.pdf Último acesso em: 30 abr. 2016.

ALBERTA LAND INSTITUTE. A guide to property rights in Alberta. Disponível em: http://www.albertalandinstitute.ca/public/download/documents/10432 Último acesso em: 30 abr. 2016.

ALBERTA TREASURY BOARD AND FINANCE. Heritage Fund - Historical Timeline. Disponível em: http://www.finance.alberta.ca/business/ahstf/history.html. Último acesso em:

BANK OF BOTSWANA. Case Study: Botswana's Management of the Pula Fund. Observance of the Santiago Principles. s/d. Disponível em: http://www.bankofbotswana.bw/assets/uploaded/BOTSWANA\%20PULA\%20FUND \%20-\%20SANTIAGO\%20PRINCIPLES\%20(2).pdf ùltimo acesso em: 03 jan. 2017 
BANCO CENTRAL DE CHILE WEBSITE. Disponível em: http://www.bcentral.cl/eng/ . Último acesso em:

BRUNEI MINISTRY OF FINANCE WEBSITE. Brunei Investment Agency. Disponível em: http://www.mof.gov.bn/English/BIA/Pages/default.aspx. Último acesso em:

CENTRAL BANK OF THE ISLAMIC REPUBLIC OF IRAN WEBSITE. Disponível em: http://www.cbi.ir/default_en.aspx. Último acesso em:

CHINA INVESTMENT CORPORATION WEBSITE. Disponível em: http://www.chinainv.cn/cicen/. Último acesso em:

DUBAI INTERNATIONAL CAPITAL WEBSITE. Disponível em: http://www.dubaiic.com/. Último acesso em:

FONDS DE RESERVE POUR LES RETRAITTES WEBSITE. Disponível em: http://www.fondsdereserve.fr/spip.php?article128. Último acesso em:

GOBIERNO BOLIVARIANO DE VENEZUELA. El Fondo de Desarollo Nacional S.A. Ministerio del Poder Popular para Economia y Finanzas. Disponível em: http://www.fonden.gob.ve/. Último acesso em:

GOVERNMENT OF ALBERTA - FINANCE AND ENTERPRISE WEBSITE. Heritage Fund Information. Disponível em: http://www.finance.alberta.ca/business/ahstf/index.html. Último acesso em:

GOVERNMENT OF SINGAPORE INVESTMENT CORPORATION WEBSITE.

Disponível em: http://www.gic.com.sg/. Último acesso em:

ISTITHMAR WORLD WEBSITE. Disponível em: http://www.istithmarworld.com/en. Último acesso em:

JEFFERIS, Keith e NEMAORAMI, Thabelo. Botswana Country Overview. Capital Resources, 2013/2014. Disponível em: http://acap.com.au/wpcontent/uploads/2013/09/BOTSWANA-COUNTRY-AND-ECONOMICOVERVIEW-2013.pdf Último acesso em: $10 \mathrm{dez} .2016$

KHAZANAH NASIONAL WEBSITE. Disponível em: http://www.khazanah.com.my/. Último acesso em: 
KINDGOM OF SAUDI ARABIA. MINISTRY OF FINANCE WEBSITE. Public Investment Fund (PTF). Disponível em: http://www.mof.gov.sa/en/docs/ests/sub_invbox.htm. Último acesso em:

KINGDOM HOLDING COMPANY (KHC) WEBSITE. Disponível em: http://www.kingdom.com.sa. Último acesso em:

KIRIBATI MINISTRY OF FINANCE AND ECONOMIC DEVELOPMENT WEBSITE. Disponível em: http://www.mfep.gov.ki/. Último acesso em:

KOREA INVESTMENT CORPORATION WEBSITE. Disponível em http://www.kic.go.kr/en/?mid=co01. Último acesso em:

KUWAIT INVESTMENT AUTHORITY OFFICIAL WEBSITE. Disponível em: http://www.kia.gov.kw/En/Pages/default.aspx. Último acesso em:

LIBYAN INVESTMENT AUTHORITY WEBSITE. Disponível em: http://www.lia.ly/. Último acesso em:

MINISTÉRIO DAS FINANÇAS DO CHILE. Relatório anual dos Fundos Soberanos, 2014. 2015. Disponível em: http://www.hacienda.cl/documento/descargar/id/14561

MINISTÉRIO DAS FINANÇAS DO CHILE. Política de investimento do FEES. s/a. Disponível em: http://www.hacienda.cl/fondos-soberanos/fondo-de-estabilizacioneconomica-y/politica-de-inversion/pauta-de-inversion.html Último acesso em: $30 \mathrm{abr}$. 2016.

MINISTÉRIO DAS FINANÇAS DA NORUEGA. Report $n^{\circ} 29$ to the Storting. March 29th, 2011. Disponível em: https://www.regjeringen.no/globalassets/upload/kilde/fin/red/2005/0013/ddd/pdfv/260 472-pmk_rap.pdf Último acesso em: 30 jan. 2016.

MINISTRY OF FINANCE OF THE RUSSIAN FEDERATION WEBSITE. Stabilization Fund of the Russian Federation. Disponível em: http://www1.minfin.ru/en/stabfund/. Último acesso em: 30 abr. 2016.

MUBADALA WEBSITE. Disponível em: http://www.mubadala.com/. Último acesso em: 
NORGES BANK WEBSITE. Disponível em: 30 jan.

2016.http://www.norgesbank.no/default 25991.aspx. Último acesso em: 30 jan. 2016.

NORDISH GOVERNMENT. Market Value of the Government Pension Fund. Disponível em: https://www.regjeringen.no/en/topics/the-economy/the-government-pensionfund/market-value-of-the-government-pension-f/id699635/ Último acesso em:

NORGES BANK INVESTMENT MANAGEMENT. Annual Report, 2014. Disponível em: http://www.nbim.no/contentassets/0ff9cd1d5c8e4737a7b7262d3ec167d4/norgesbank-investment-management-annual-report-20141.pdf Último acesso em:

NORWEGIAN PETROLEUM DIRECTORATE. Facts, 2014. Disponível em: http://www.npd.no/Global/Engelsk/3Publications/Facts/Facts2014/Facts_2014_nett_pdf Último acesso em:

QATAR INVESTMENT AUTHORITY WEBSITE. Disponível em: http://www.qia.qa/qia/index.html Último acesso em: 30 jan. 2016.

QUEENSLAND INVESTMENT CORPORATION (QIC) WEBSITE. Disponível em: http://www.qic.com.au/default.aspx. Último acesso em:

STATE GENERAL RESERVE FUND WEBSITE. Disponível em: http://www.sgrf.gov.om/. Último acesso em: 30 jan. 2016.

STATE OIL FUND (SOFAZ) OF THE REPUBLIC OF AZERBAIJAN WEBSITE. Disponível em: http://www.oilfund.az/en. Último acesso em: 30 jan. 2016.

STATISTICS NORWAY. Pirâmide Etária da População Norueguesa. Disponível em: https://www.ssb.no/en/befolkning/statistikker/folkemengde/_image/218947.png?_enco $\underline{\text { ded }=2 \mathrm{f} 66666666666678302 \mathrm{f} 35382 \mathrm{f} 29303136286874646977656 \mathrm{c} 616373 \& \text { ts }=14 \mathrm{~b} 9 \mathrm{c}}$ $\underline{3 \mathrm{a} 668}$

TEMASEK HOLDINGS WEBSITE. Disponível em: http://www.temasekholdings.com.sg/ Último acesso em: 30 jan. 2016.

THE WORLD BANK. GINI index. Disponível em: http://data.worldbank.org/indicator/SI.POV.GINI?locations=BW Último acesso em: 10 dez. 2016. 
Botswana overview. Disponível em:

http://www.worldbank.org/en/country/botswana/overview Último acesso em: 10 dez. 2016.

THE HERITAGE FOUNDATION. Botswana Economic Freedom Score. Index of Economic Freedom, 2016. Disponível em:

http://www.heritage.org/index/pdf/2016/countries/botswana.pdf Último acesso em: 10 dez. 2016.

UNITED STATES ENERGY INFORMATION ADMINISTRATION. Norway.

Disponível em: http://www.eia.gov/beta/international/country.cfm?iso=NOR Último acesso em: 01 abr. 2015.

\section{Lista de FSR do SWF - Sovereign Wealth Funds Institute}

ABU DHABI INVESTMENT AUTHORITY. List of Sovereign Wealth Funds. Wealth Fund Institute, 2010. Disponível em: http://www.swfinstitute.org/fund/adia.php

ALGERIA - REVENUE REGULATION FUND. List of Sovereign Wealth Funds. Wealth Fund Institute, 2010. Disponível em: http://www.swfinstitute.org/fund/algeria.php.

AUSTRALIAN FUTURE FUND. List of Sovereign Wealth Funds. Wealth Fund Institute, 2010. Disponível em: http://www.swfinstitute.org/fund/australia.php.

AZERBAIJAN - STATE OIL FUND. List of Sovereign Wealth Funds. Wealth Fund Institute, 2010. Disponível em: http://www.swfinstitute.org/fund/azerbaijan.php.

BRUNEI INVESTMENT AGENCY. List of Sovereign Wealth Funds. Wealth Fund Institute, 2010. Disponível em: http://www.swfinstitute.org/fund/brunei.php

CANADA - ALBERTA'S HERITAGE FUND. List of Sovereign Wealth Funds. Wealth Fund Institute, 2010. Disponível em: http://www.swfinstitute.org/fund/alberta.php

CHILE - PR \& ES FUND. List of Sovereign Wealth Funds. Wealth Fund Institute, 2010. Disponível em: http://www.swfinstitute.org/fund/chile.php

CHINA INVESTMENT CORPORATION. List of Sovereign Wealth Funds. Wealth Fund Institute, 2010. Disponível em: http://www.swfinstitute.org/fund/cic.php 
FRANCE - STRATEGIC INVESTMENT FUND. List of Sovereign Wealth Funds. Wealth Fund Institute, 2010. Disponível em: http://www.swfinstitute.org/fund/france.php IRAN - OIL STABILISATION FUND. List of Sovereign Wealth Funds. Wealth Fund Institute, 2010. Disponível em: http://www.swfinstitute.org/fund/iran.php KAZAKHSTAN NATIONAL FUND. List of Sovereign Wealth Funds. Wealth Fund Institute, 2010. Disponível em: http://www.swfinstitute.org/fund/kazakhstan.php KIRIBATI - REVENUE EQUALIZATION RESERVE FUND. List of Sovereign Wealth Funds. Wealth Fund Institute, 2010. Disponível em: http://www.swfinstitute.org/fund/kiribati.php

KOREA INVESTMENT CORPORATION. List of Sovereign Wealth Funds. Wealth Fund Institute, 2010. Disponível em: http://www.swfinstitute.org/fund/korea.php KUWAIT INVESTMENT AUTHORITY. List of Sovereign Wealth Funds. Wealth Fund Institute, 2010. Disponível em: http://www.swfinstitute.org/fund/kuwait.php

LIBYAN INVESTMENT AUTHORITY. List of Sovereign Wealth Funds. Wealth Fund Institute, 2010. Disponível em: http://www.swfinstitute.org/fund/libya.php MALAYSIA - KHAZANAH NASIONAL. List of Sovereign Wealth Funds. Wealth Fund Institute, 2010. Disponível em: http://www.swfinstitute.org/fund/malaysia.php

NORWAY - GOVERNMENT PENSION FUND - GLOBAL. List of Sovereign Wealth Funds. Wealth Fund Institute, 2010. Disponível em: http://www.swfinstitute.org/fund/norway.php

OMAN - STATE GENERAL RESERVE FUND. List of Sovereign Wealth Funds. Wealth Fund Institute, 2010. Disponível em: http://www.swfinstitute.org/fund/oman.php

QATAR INVESTMENT AUTHORITY. List of Sovereign Wealth Funds. Wealth Fund Institute, 2010. Disponível em: http://www.swfinstitute.org/fund/qatar.php

SAUDI ARABIA - PUBLIC INVESTMENT FUND. List of Sovereign Wealth Funds. Wealth Fund Institute, 2010. Disponível em: http://www.swfinstitute.org/fund/saudipif.php SINGAPORE - GIC. Disponível em: http://www.swfinstitute.org/fund/gic.php 
SINGAPORE - TEMASEK HOLDINGS. List of Sovereign Wealth Funds. Wealth Fund Institute, 2010. Disponível em: http://www.swfinstitute.org/fund/temasek.php UAE - INVESTMENT CORPORATION OF DUBAI. List of Sovereign Wealth Funds. Wealth Fund Institute, 2010. Disponível em: http://www.swfinstitute.org/fund/dubai.php

UAE - MUBADALA DEVELOPMENT COMPANY. List of Sovereign Wealth Funds. Wealth Fund Institute, 2010. Disponível em: http://www.swfinstitute.org/fund/mubadala.php

USA - ALASKA PERMANENT FUND. List of Sovereign Wealth Funds. Wealth Fund Institute, 2010. Disponível em: http://www.swfinstitute.org/fund/alaska.php 